\title{
Deformation induced fluid flow behaviour of subglottic mucosa
}

\begin{abstract}
We explore the effect of deformation induced flow behaviour within the subglottic region of human larynx using a computational fluid dynamic framework and simplified subglottal space obtained from computed tomography imaging of the human larynx. An experimental investigation, conducted by the authors, to determine the stress/ strain behaviour of mucosa using 6-donor human larynges suggested the variation of its elasticity in the subglottic region as a function of distance from vocal fold. The computed tomography imaging of human larynx was utilised to approximate the geometry of subglottic internal space which is used to investigate the effect of deformation of subglottic mucosa on the air flow during the myoelastic cycle. A parametric study was conducted to simulate a laminar flow within a subglottic space using computational fluid dynamics with appropriate numerical approximation and the choice of boundary conditions and their implementation is discussed. It is shown that the deformation of subglottic mucosa plays a significant role in the delivery of low-pressure air flow over the vocal folds and helps to maintain laminar flow due to venturi effects. Limitations of this study and detailed suggestions for investigation and future implementation are provided.
\end{abstract}

Keywords: vocal fold, phonation, biomechanics, tissue deformation, phonation
Volume I Issue 6 - 2017

\author{
Hafiz Anwar, ${ }^{1,2}$ Eric Goodyer, ${ }^{3}$ Frank Muller, ${ }^{4}$ \\ Markus Hess, ${ }^{4}$ Farukh Farukh, ${ }^{5}$ Karthikeyan \\ Kandan ${ }^{5}$ \\ 'Faculty of Sciences, the University of Nottingham, UK \\ ${ }^{2}$ Department of Mechanical Engineering, Institute of Space \\ Technology, Pakistan \\ ${ }^{3}$ School of Computer Science, De Montfort University, UK \\ ${ }^{4}$ Universitat Klinic, Germany \\ ${ }^{5}$ School of Engineering \& Sustainable Development, De Montfort \\ University, UK
}

Correspondence: Farukh Farukh, School of Engineering \& Sustainable Development, Faculty of Technology, De Montfort University, UK, Tel +44 I |62577028, Email F.Farukh@dmu.ac.uk

Received: December 16, 2017 | Published: December 27, 2017
Abbreviations: LSR, linear skin rheometer; CFD, computational fluid dynamics; SCI, scientific computing and imaging; SIMPLE, semi implicit method for pressure linked equations

\section{Introduction}

It is well known that both subglottal and supraglottal spaces play an important role during phonation. Supraglottal region being easy to access is studied by many researchers and understood well whereas subglottal space is not well understood due to the difficulty in visualisation. Various studies suggested the importance of subglottic region in phonation. According to Wyke \& Kirchner, ${ }^{1}$ subglottal mucosa contributes to the reflex control of the intrinsic laryngeal muscles during phonation. Sundberg ${ }^{2}$ found that mechanoreceptors are present in the subglottal mucosa that plays a role in phonation. Smith $^{3}$ identified that resection in this region affects phonation. This suggests that the subglottis is not merely a 'pipe' that delivers airflow over the vocal folds, but play a significant role in phonation. This paper deals with a numerical model to gain a better understanding of the way this region functions during phonation. The focus of this study is to examine the biomechanics and deformation of mucosal tissues in this region and its impact on the aerodynamics of the airflow over the vocal folds using simplified subglottal model.

In the recent decade, development of the computational modelling techniques has greatly enhanced our understanding of human phonation. ${ }^{4}$ Detailed glottal flow fields have been revealed by the researchers. For example, a strong interaction between the glottal jet and supraglottal wall was presented by the authors. Xue Q et al. ${ }^{5}$ Zheng $\mathrm{X}$ et al. ${ }^{6}$ Qian et al. ${ }^{7}$ suggested the complex shapes of the larynx will produce a profound effect of the flow and used the realistic patient specific lyranx geometry to investigate the human phonation. However, all these studies considered the subglottal part as a non- deformable region which may not be the case in reality and thus the phonation remains elusive. The variation of mechanical properties of subglottic mucosa in animals as a function of its distance from the vocal fold was studied in the literature. ${ }^{6,7}$ In order to determine the tissue structure of this region in human, six human larynges were studied by the authors Goodyer E \& Müller F. ${ }^{8}$ It was observed that human larynx presents similar variations in elasticity as that observed in animals. It provides the basis for our hypothesis that sub-glottal region has deformation characteristics. In order to establish the importance of considering the deformation behaviour of the subglottal region and its impact on the aerodynamics of the airflow over the vocal folds is studied.

\section{Research hypothesis and objectives}

A hypothesis that led to this study is based on a detailed experimental characterization of elasticity gradient in the subglottic region, given elsewhere. ${ }^{8}$ The main results are briefly recalled here for the sake of completness, as they will help to understand the reason for this study. Using data obtained from six donor larynges, a series of measurements of the tissue elasticity were taken using a Linear Skin Rheometer (LSR). The LSR delivered a controlled cyclical force of $\pm 1 \mathrm{~g}$ to the attachment point and the displacement was logged. The stress/strain characteristics in terms of grams force per millimetre displacement are obtained by shearing the surface in the direction of airflow inferior to superior normalised with respect to the measured tissue elasticity of the vocal folds. As can be seen in Figure 1, the mucosa near to the vocal folds exhibit a similar elastic modulus, with stiffness increasing as the measurements are taken in the inferior direction

Following this experimental observation, it is hypothesised that the variance in elasticity of the mucosa in the sub-glottal region will result in a deformation characteristic that forms a more gradual 
narrowing of the airflow path during uplift of the vocal folds. This tendency to create a more gradual slope will result in a reduction in turbulence and enhance laminar flow. Oren et al. ${ }^{9}$ concluded that "the smooth converging shape of the sub-glottis can produce a significant reduction in turbulence", which also aligns with fluid dynamics theory. To investigate this hypothesis in full, a series of studies are required, covering both the physiological aspects including biomechanics and the myoelastic cycle, and the synthesis of a full 3D model to investigate the change in the aerodynamics of the anatomical structures during deformation. However, as an initial study, a twodimensional bio-mechanical flow simulation using a simplified subglottic space is presented here. The results from this study help to not only to verify the hypothesis but to provide the basis for further investigation.

\section{Anatomical model and simulation setup}

In order to get the approximate geometry for the CFD analysis, a thin-slice CTscan (1 $\mathrm{mm}$ plane-to-plane resolution) was performed on a larynx sample obtained from a mortuary case. A sample image of a 3D reconstruct obtained from this CT scan using open-source medical imaging software-Seg3D2 (Developed by the NIH Center for Integrative Biomedical Computing at the University of Utah Scientific Computing and Imaging (SCI) Institute) is shown in Figure 2. This thin-slice CT scan provides sufficient resolution to approximate the air flow path of sub-glottal region. The outline of the airway shown in Figure 2 was then simplified and used for the computational analysis. The approximate dimensions of the geometry are $20 \mathrm{~mm}$ width at inlet, $10 \mathrm{~mm}$ width at outlet (before deformation) and $40 \mathrm{~mm}$ length of the subglottal region as shown in Figure 3a. In order to understand the effect of conic deformation on airflow, the outlet was reduced to a size of $7.5 \mathrm{~mm}$ in simulations. To support our theory we analysed the airflow through CFD of an idealised sub-glottic jet, one being $1 \mathrm{~cm}$ in diameter, which is typical for a male, and the other being $0.75 \mathrm{~cm}$ in diameter being typical for a female. The airflow was modelled at STP conditions. The non-slip-non-penetration flow condition is applied on all of the walls. In order to simulate the air flow, 0 and $1.2 \mathrm{kPa}$ gauge pressure are applied at the outlet and inlet, respectively. In this study, the Navier-Stokes equations were solved using SIMPLE (Semi-Implicit Method for Pressure Linked Equations) algorithm. The software package used for this purpose is ANSYS-Fluent 16.0. The second order upwind scheme is used to analyze the grids. The flow solver employs a high-resolution, non-uniform 124176 Cartesian grid for the computational domain.

\section{Results}

Linear Skin Rheometer (LSR) data obtained from 6 donor larynges is shown in Figure 1 as mean of the normalized results. As can be seen in this figure, mucosa near to the vocal folds exhibits a similar elastic modulus, with stiffness increasing as the measurements are taken in the inferior direction. Trend line in Figure 1 indicates that stiffness increased by approximately $0.058 \mathrm{~g} / \mathrm{mm}$ for a displacement of $1 \mathrm{~mm}$. The temporal and spatial distribution of aerodynamic pressure and velocity results obtained from CFD simulation are given in Figure 3 for all the simplified sub-glottal geometries. In addition, it shows the results considering different deformation of the subglottal region. As can be seen in Figure 3 that the pressure contours within the constriction the flow is laminar. Besides, an increase in air velocity is observed towards the opening also point towards laminar flow. Thus, the hypothesis that the deformation of the subglottal mucosa will reduce the occurrence of vortices and enhance laminar flow is verified. This is the initial release of our finding. Future work will include a large scale study to obtain a more precise map of the variance of elasticity over the mucosa and the determination of the degree of anisotropic behaviour. To advance the state of the art, a full scale 3D model based on realistic geometric reconstruction of both the larynx and airway will be required for this purpose. It will be obtained using the technique mentioned in section 3 . The 3D models will be enhanced to include a time-series showing the change in the structure during uplift caused by the variable deformation of the mucosa. Finally standard Computational Fluid Dynamics (CFD) analysis will be carried out to model the effect of conic deformation on airflow inferior of the vocal folds using realistic subject-specific larynx.

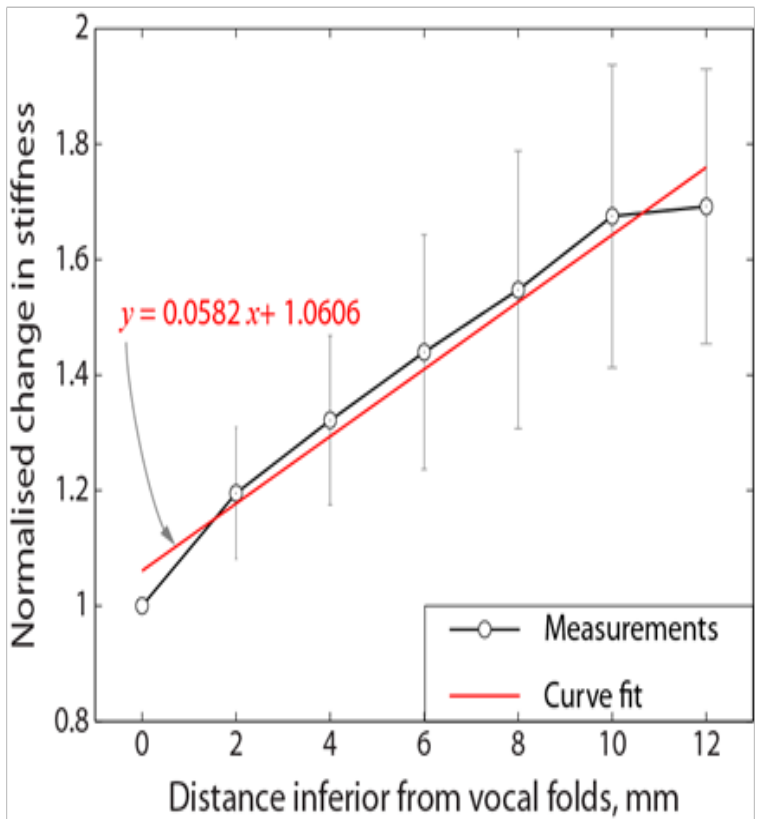

Figure I The measured mean normalized change in mucosa stiffness as a function of distance inferior from the vocal folds. The circles indicate the measured normalized data taken at a specific location measured from all 12 hemi-larynges investigated in this study.

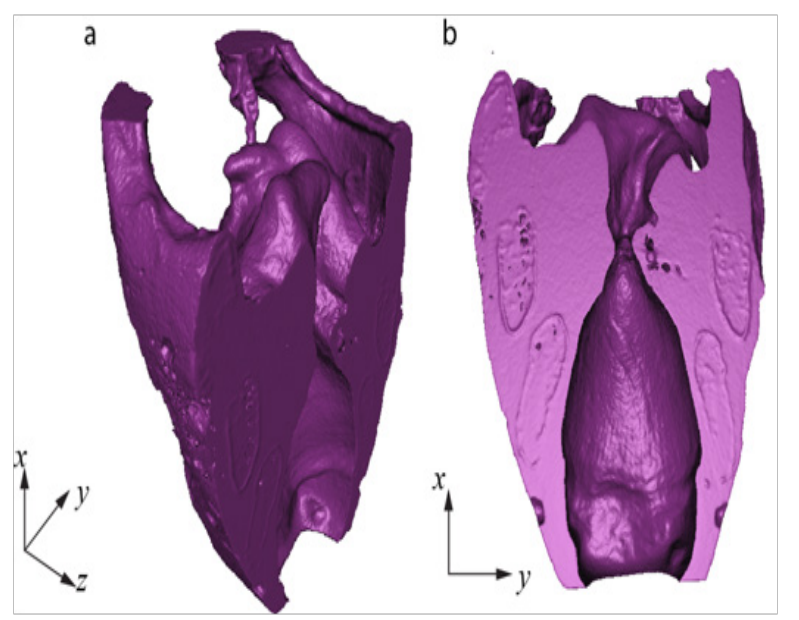

Figure 2 Three-dimensional (3D) model of a larynx constructed from a computed tomography scan images $(A)$ showing isometric view of a sliced 3D model and (B) cross-section highlighting the conical geometry of the larynx along the sectioned-plane. 
a
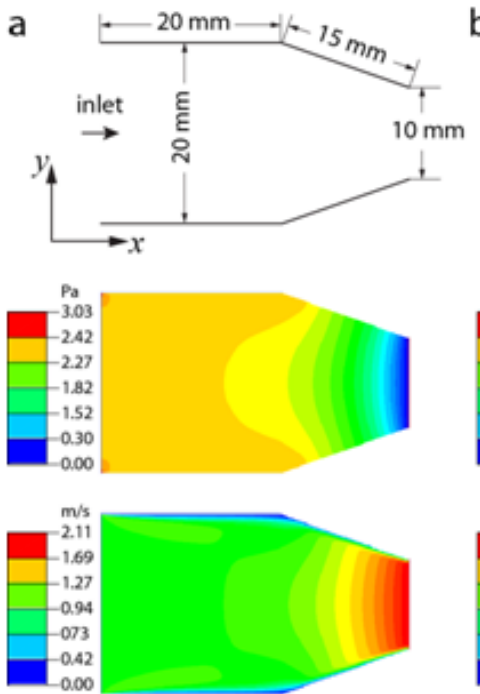

b

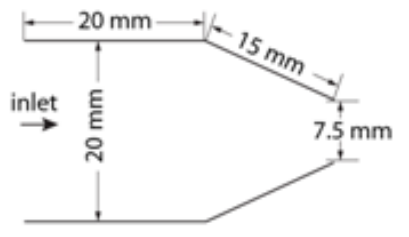

C
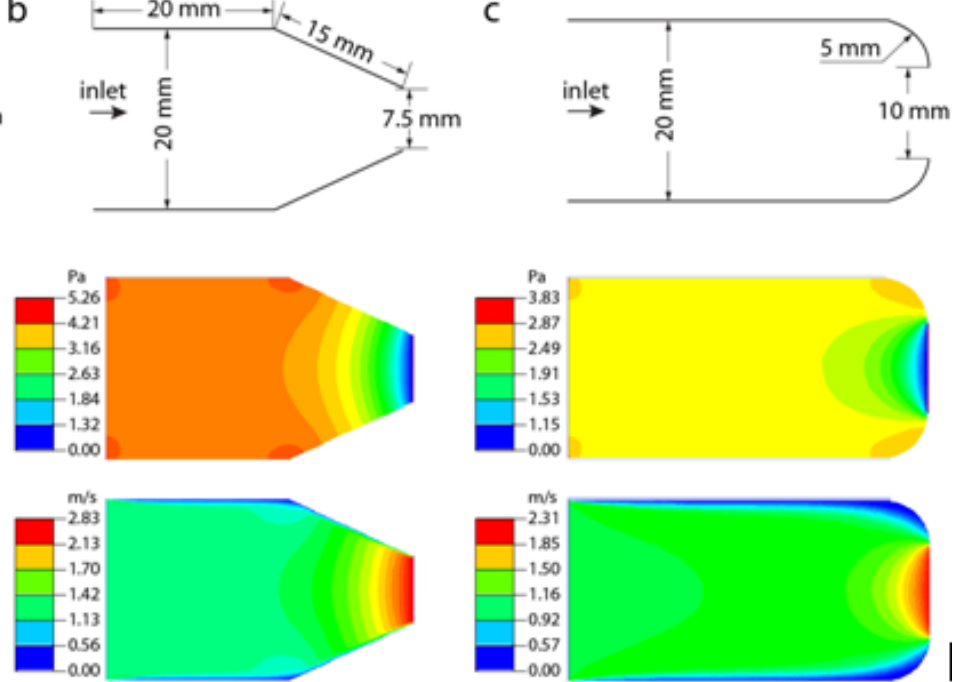
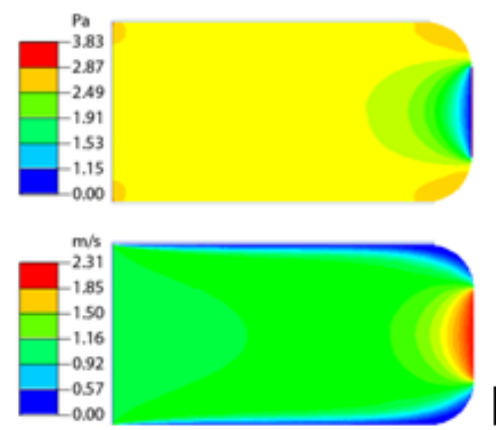

Figure 3 Two-dimensional computational fluid dynamics calculations illustrating pressure drop and increase in velocity for (A) $10 \mathrm{~mm}$, (B) $7.5 \mathrm{~mm}$ and (C) slittype opening suggesting laminar flow.

\section{Conclusion}

This study indicates that under low pressure conditions the subglottic mucosa will deform to form a conic shape that is a gradual narrowing of the airflow path. Initial simplified models of the impact on the aerodynamics through such a construction support the maintenance of laminar flow. A full scale 3D model based on actual anatomical structures that use elasticity data to create a time-series of the change in shape due to deformation will now be investigated. It will be followed by an investigation of the impact of this deformation on the aerodynamics using full scale 3D model. This model will be an important precursor step towards development of a computer tool to diagnose voice disorders.

\section{Acknowledgements}

This research was supported by the Engineering Physics and Science Research Council (EPSRC) of the United Kingdom (EP/ N018931/1).

\section{Conflict of interest}

The author declares that there is no conflict of interest.

\section{References}

1. Wyke B, Kirchner J. Neurology of the larynx. In: Hinchcliffe R, Harrison D. editors. Scientific foundation of otolaryngology. UK: William Heinemann Medical Books;1976. p. 546-566.
2. Sundberg J, Iwarsson J, Billström AH. Significance of mechanoreceptors in the subglottal mucosa for subglottal pressure control in singers. $J$ Voice. 1995;9(1):20-26.

3. Smith ME, Roy N, Stoddard K. How does cricotracheal resection affect the female voice? Ann Oto Rhino Laryngology. 2008;117(2):85-89.

4. Mittal R, Iaccarino G. Immersed boundary methods. Annu Rev Fluid Mech. 2005;37:239-261.

5. Xue Q, Mittal R, Zheng X, et al. Computational modeling of phonatory dynamics in a tubular three-dimensional model of the human larynx. $J$ Acoust Soc Am. 2012;132(3):1602-1613.

6. Zheng X, Mittal R, Xue Q, et al. Direct numerical simulation of glottal jet and vocal fold dynamics in a three dimensional laryngeal model. $J$ Acoust Soc Am. 2011;130(1):404-415.

7. Xue Q, Zheng X. Subject-specific computational modeling of human phonation. J Acoust Soc Am. 2014;135(3):1445-1456.

8. Goodyer E, Müller F, Hess M, et al. Biomechanical Flow Amplification Arising From the Variable Deformation of the Subglottic Mucosa. $J$ Voice. 2017;31(6):669-674.

9. Oren L, Khosla S, Shanmugam MD, et al. Role of Subglottal Shape in Turbulence Reduction Annals of Otology. Rhinology \& Laryngology. 2009;118(3):232-240. 